RR-73-12

NONSTATIONARITY AND PORTFOLIO CHOICE

Christopher B. Barry

Robert L. Winkler

November 1973

Research Reports are publications reporting on the work of the author. Any views or conclusions are those of the author, and do not necessarily reflect those of IIASA. 


\title{
Nonstationarity and Portfolio Choice
}

\author{
Christopher B. Barry* and Robert L. Winkler**
}

\section{Abstract}

In this paper some effects of nonstationary parameters upon inferences and decisions in portfolio analysis are investigated. A Bayesian inferential model with nonstationary parameters is presented and is applied to the problem of portfolio choice. For this model, nonstationarity l) implies greater uncertainty about future returns; 2) implies that in forecasting future returns, recent returns should receive more weight than not-so-recent returns; 3 ) restricts the amount of information that can be obtained about future values of the parameters of interest; 4) shifts investment among risky securities and from risky securities to risk-free securities; and 5) yields optimal portfolios with smaller expected returns than corresponding optimal portfolios in the stationary case.

\section{Introduction}

Formal models for decision making under uncertainty generally represent the uncertainty facing the decision maker in terms of probability distributions. One element of economic reality--change--has been consistently ignored in many areas for which formal models for decision making have been developed. That is, most models have assumed stationary probability distributions in what appears to be a nonstationary world. ${ }^{1}$

* College of Business Administration, University of Florida, U.S.A.

* Graduate School of Business, Indiana University, U.S.A.; research scholar at the International Institute for Applied Systems Analysis. 
One area in which Eormal models have received a great deal of interest but in which change has been persistently overlooked is portfolio analysis. In the work of Markowitz [2], which laid the foundation for most of the formal portfolio models that are studied today, and in the single-period models that followed (many of which are summarized in Francis and Archer [3]), no mention was made of nonstationarity. In addition, multiperiod models (e.g. Tobin [4], Mossin [5], Chen, Jen, and Zionts [6], and Hakansson [7]) have not explicitly considered the effects of nonstationarity. General models of consumption and irryestment have been developed (e.g. Ilakarsson [1], Samuelson [9], Fama [10], and Meyer [11]), and again nonstationarity has not been directly confronted.

I'the ecoiomic arguments for the existence of nonstatiorarity in stock price distributions are straightforward. The price behavior of particular securities is affected by characteristics of the firm (e.g. capital structure, marketing strategies, product lines, top management, etc.) as well as by the general economic environment in which the firm operates (e.g. aspects of the econoiny as a whole and characteristics of the competitors, suppliers, and customers of the firm). As characteristics of a given firm and the economic environment change over time, the anticipated profits of the firm may change, and the valuation of the firm's securities by the stock market may be affected.

Several recent studies provide empirical support for the claim that the parameters of distributions of stock-price- 
related variables may change over time. In a study using the stable paretian model for the distribution of log price relatives, Barnea and Downes [12] found that the stable distributions that appeared to explain their data exhibited nonstationary parameters. Praetz [13] found a good fit for stock index data using a $t$ distribution, which is consistent with returns that are normally distributed with shifting variance, and study by Blattberg and Gonedes [14] amplified considerably the findings of Praetz. Boness, Chen, and Jatusipitak [15] suggested that log price changes are normally distributed with discrete shifts in variance; firms were studied before and after capital structure changes, and the log price differences were found to have different variances in the two periods. Hsu, Miller, and Wichern [16] obtained similar results and arrived at the following conclusion $[16, p .113]$ :

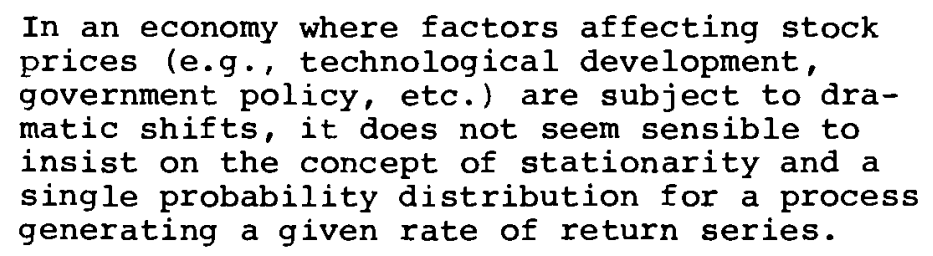

This paper is concerned with the effects of nonstationarity on portfolio decisions. A convenient framework for studying the problem of changing parameters, both in terms of forecasting security prices and in terms of portfolio decision making, is provided by the Bayesian approach to statistical inference and decision. Winkler [17] recently developed a Bayesian model for forecasting future security prices, 
and an extension of that model to the nonstationary case is discussed in Section 2 of this paper. Some recent results concerning nonstationary means in a multinormal process are utilized to illustrate the model, and the nonstationary model is contrasted with the corresponding stationary model in terms of both short-run and limiting features. In section 3 a very brief cescription of the Bayesian portfolio selection and revision model of winkler and Barry [18] is given, and in Section 4 the nonstationary forecasting model of Section 2 is used to determine the effects of nonstationarity on portfolio decistons made with the model of section 3 . Two cases are examined in some detail, the case of two risky securities and the case of one risky security and one risk-free security. ioreover, the effects of nonstationarity on the efficient set and the optimal portiolio from a standard Markowitz-type model are also described in section 4 . Some conclusions and suggested extensions are presented in section 5 .

\section{A Bayesian Model for Forecasting Security Prices Under Nonstationarity}

In this section a model involving a multinormal datagenerating process with nonstationary mean vector will be considered. The random variables of interest are return vectors $\tilde{r}_{t}$ consisting of elements $\tilde{r}_{t}^{j}$, the return on security $j(j=1, \ldots, J)$ during $t i m e$ period $t(t=1,2, \ldots) .^{2}$ suppose that $\tilde{\sim}_{t}$ is normally distributed with unknown mean vector ${\underset{\sim}{\tilde{u}}}_{t}$ ana known positive-definite covariance matrix $\underset{\sim}{\Sigma}$. Assume that at the start of period $t$ (i.e., at time $t-1$ ), the decision 
maker has a normal prior distribution for $\underset{\sim}{\tilde{t}}$ with mean vector $\mathrm{m}_{\sim}^{\prime}$ and covariance matrix $\left(\mathrm{n}_{t}^{\prime}\right)^{-1} \sum_{\sim}$. The marginal distribution of ${\underset{\sim}{\mathrm{r}}}_{\mathrm{t}}$ at the start of period $t$ (called a predictive distribution) is then normal with mean vector $m_{t}^{\prime}$ and covariance matrix $\left[\left(n_{t}^{\prime}+1\right) / n_{t}^{\prime}\right] \sum_{\sim}$. For many decision-making purposes this distribution may be the most essential product of the analysis, and this will be the case in the application of the Bayesian model to portfolio analysis.

After ${\underset{\sim}{\mathrm{r}}}_{\mathrm{t}}$ is observed, the posterior distribution of ${\underset{\sim}{\mathrm{H}}}_{t}$ is normal ${ }^{3}$ with mean vector $m_{\sim}^{\prime \prime}$ and covariance matrix $\left(n_{t}^{\prime \prime}\right)^{-1}{\underset{\sim}{\sim}}_{\text {, }}$ where

$$
n_{t}^{\prime \prime}=n_{t}^{\prime}+1
$$

and

$$
m_{t}^{\prime \prime}=\frac{n_{t \sim t}^{\prime} m_{\sim}^{\prime}+\underset{\sim t}{r}}{n_{t}^{\prime}+1} .
$$

Next, assume that successive mean vectors satisfy

$$
\tilde{\sim}_{t+1}=\tilde{\sim}_{t}+\tilde{\sim}_{t},
$$

where $\underset{\sim}{\tilde{\varepsilon}}$ is normally distributed with mean vector $\underset{\sim}{e}$ and covariance matrix $\omega^{-1}{\underset{\sim}{\Sigma}}_{,}$and ${\underset{\sim}{\tau}}_{t}$ and $\tilde{\sim}_{t}$ are independent. Transforming the posterior distribution of $\underset{\sim}{\tilde{\mu}}$ into a prior distribution of $\tilde{\sim}_{t+1}$ yields a normal distribution with mean vector

$$
\underset{\sim t+1}{m_{t}^{\prime}}=\underset{\sim t}{m_{t}^{\prime \prime}}+\underset{\sim}{e}
$$


and covariance matrix $\left(n_{t+1}^{\prime}\right)^{-1} \sum_{\sim}$, where

$$
n_{t+1}^{\prime}=\left[\left(n_{t}^{\prime}+1\right)^{-1}+w^{-1}\right]^{-1}
$$

(see [20]). In this manner, a sequence of prior and posterior distributions for successive $\tilde{\sim}_{t}$ may be obtained as successive $\tilde{r}_{t}$ are observed.

Under stationarity, $\underset{\sim}{\underline{\underline{\varepsilon}}}=\underset{\sim}{\mathrm{o}}$ for all $t$, and $\underset{\sim 1}{\tilde{\mu}}=\tilde{\sim}_{2}=\cdots$ $=\underset{\sim}{\tilde{\mu}}=\cdots=\underset{\sim}{\tilde{\mu}}$. Thus, $\underset{\sim}{e}=0$ and $\omega^{-1}=0$, in which case (4) and (5) simplify to $\underset{\sim}{m_{t+1}^{\prime}}={\underset{\sim}{m}}_{t}^{\prime \prime}$ and $n_{t+1}^{\prime}=n_{t}^{\prime}+1$. Under stationarity, then, the prior distribution of ${\underset{\sim}{\tilde{\mu}}}_{t+1}$ at the start of period $t+1$ is the same as the posterior distribution of $\tilde{\sim}_{t}$ at the end of period $t$. Note that if $\omega^{-1}>0, n_{t}^{\prime}+1$ is clearly greater than the right-hand side of (5). Thus, $\mathbf{n}_{t+1}^{\prime}$ is greater under stationarity than under nonstationarity, given that $n_{t}^{\prime}$ is the same in the two cases.

In the case of nonstationarity with no $\operatorname{drift}^{4} \underset{\sim}{\mathrm{e}}=\underset{\sim}{0}$. Thus, for a given posterior distribution of $\tilde{\sim}_{t}$ at time $t$, the only difference between the prior distributions of ${\underset{\sim}{\tilde{\mu}}}_{t+1}$ under stationarity vis-a-vis nonstationarity with no drift is that the term $n_{t+1}^{\prime}$ is larger in the former case.

The covariance matrix of the predictive distribution of $\underset{\sim}{\tilde{r}_{t+1}}$ at time $t$ is $k_{t+1} \sum_{\sim}$, where $k_{t+1}=\left(n_{t+1}^{\prime}+1\right) / n_{t+1}^{\prime}$. Furthermore, an investment portfolio comprised of the $\mathrm{J}$ securities may be described by the $J \times 1$ vector $\underset{\sim}{a}$, where the $j$ th element of $a$ is the dollar value of investment in security $j$, and the predictive variance of the value of the portfolio at time $t+1$ is given by the quadratic form $k_{t+1} \underset{\sim}{a} \underset{\sim}{T_{\sim}} \underset{\sim}{ }$. But $k_{t+1}$ 
is larger under nonstationarity than under stationarity (because $n_{t+1}^{\prime}$ is smaller under nonstationarity), and $\sum_{\sim}$ is positive definite. Thus, the portfolio variance is greater under nonstationarity, provided that $\underset{\sim}{a} \neq \underset{\sim}{0}$, of course. With normal distributions of returns and a risk-averse investor, this implies that any given portfolio is properly viewed as riskier under nonstationarity than under stationarity. ${ }^{5}$ This should not be surprising, since past observations of returns provide relatively less information about the current value of $\underset{\sim}{\tilde{\mu}}$ under nonstationarity than under stationarity. Less information implies greater uncertainty, which should be reflected by an increase in the measure of uncertainty, variance. Therefore, considering either a single risky security or a portfolio of securities, nonstationarity implies greater uncertainty. ${ }^{6}$

Next, consider the limiting behavior of successive prior distributions. In the case of stationary means,

$$
n_{t}^{\prime}=n_{1}^{\prime}+t-1 \text {. }
$$

Obviously, as $t$ increases, $n_{t}^{\prime}$ increases without bound. Since the covariance matrix of the prior distribution of $\underset{\sim}{\tilde{\mu}}\left(=\sim_{\sim}^{\tilde{\mu}}\right.$ for all $t)$ is $\left(n_{t}^{\prime}\right)^{-1} \underbrace{}_{\sim}$, it follows that the covariance matrix tends to $\stackrel{\sim}{\circ}$ (a J $x$ J matrix of zeros).

With nonstationary means, successive values of $n_{t}^{\prime}$ are computed from (2.5). For $n_{i}^{\prime}>0, n_{t}^{\prime}$ converges to the limit

$$
n_{L} \equiv \lim _{t \rightarrow \infty} n_{t}^{\prime}=\left[(1+4 \omega)^{1 / 2}-1\right] / 2,
$$


and the convergence is monotone (see [20]). Therefore, the covariance matrix of the prior distribution of $\underset{\sim}{\tilde{\mu}}$ converges to $\left(n_{L}\right)^{-1} \sum_{\sim}$. This implies the intuitively appealing result that uncertainty about $\tilde{\sim}_{t}$ cannot be reduced indefinitely under the prescribed conditions of nonstationarity. In fact, if $n_{i}^{\prime}>n_{L},\left\{n_{t}^{\prime}\right\}$ will be a decreasing sequence, so that successive prior distributions will reflect increasing uncertainty about their respective arguments $\tilde{\sim}_{t}$. The inability to eliminate uncertainty about $\underset{\sim}{\tilde{\sim}}$ as $\underset{\sim}{\tilde{\sim}}$ shifts and additional returns are observed is of course a product of the stochastic nature of the shocks $\tilde{\varepsilon}_{t}$, and $n_{L}$ is a decreasing function of $\omega^{-1}$, the scalar factor in the covariance matrix of $\tilde{\varepsilon}_{t}$.

Because the shocks are stochastic, the relevance of any observed return, say $\underset{\sim}{r} t-i$, for making inferences about a later value of the mean vector, say $\tilde{\mu}_{t+1}$, decreases as $i$ increases. In particular, the weight assigned to the return

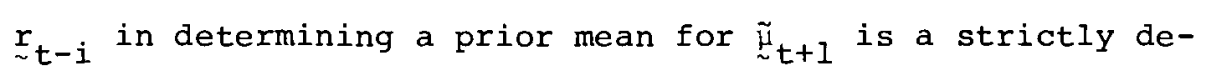
creasing function of $i$ (see [20]). In the special case in which $n_{I}^{\prime}=n_{L}$, for example, $n_{t}^{\prime}=n_{L}$ for all $t \geq 1$, and the prior mean of ${\underset{\sim}{\tilde{u}}}_{t+1}$ at the start of period $t+1$ can be expressed in the form

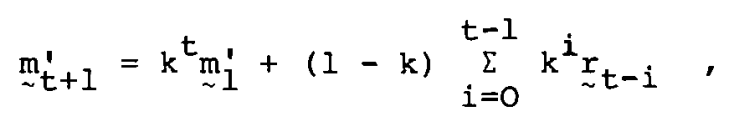

where

$$
k=n_{L} /\left(n_{L}+I\right) \text {. }
$$


Here $k$ is between zero and one, and the weight of $\underset{\sim t-i}{r}$ is $(1-k) k^{i}$, an exponentially decreasing function of $i$. Note that $1-k$, the weight given to the most recent return, is a decreasing function of $\mathrm{n}_{\mathrm{L}}$ (hence an increasing function of $\left.\omega^{-1}\right)$; the greater the degree of nonstationarity, as measured by the scalar factor of the covariance matrix of $\underset{\sim}{\tilde{\varepsilon}_{t}}$, the less weight is given to all but the most recent return. This provides an indication of the decreasing relevance of a given price difference in one period for making inferences about values of $\tilde{\sim}_{t}$ further and further into the future. In contrast, all of the past returns are weighted equally under stationarity, and $\underset{\sim t+1}{m}$ can be expressed in the form

$$
\underset{\sim t+1}{m_{t}^{\prime}}=\frac{n_{1 \sim 1}^{\prime} m_{i}+\sum_{i}^{t}{\underset{\sim}{i}}_{n}^{r}}{n_{1}^{\prime}+t} .
$$

One possible extension of the model presented here would be the consideration of nonstationary variance and covariance terms. To generalize the approach of this section yet further, replace $\underset{\sim}{\tilde{r}}$ by $\tilde{\sim}_{t}$ ' which can represent any variables of interest, and replace $\tilde{\sim}_{t}$ by $\tilde{\theta}_{t}$. The data-generating process of interest can then be represented by $f\left(\underset{\sim}{x} \mid \theta_{t}\right)$. The prior distribution of $\tilde{\theta}_{t}$ at the start of period $t$ is $f^{\prime}\left(\theta_{t}\right)$, and the posterior distribution $f$ " $\left({\underset{\sim}{\theta}}_{t} \mid \underset{\sim}{\mathbf{x}_{t}}\right)$ can be obtained once $\mathbf{x}_{t}$ is observed:

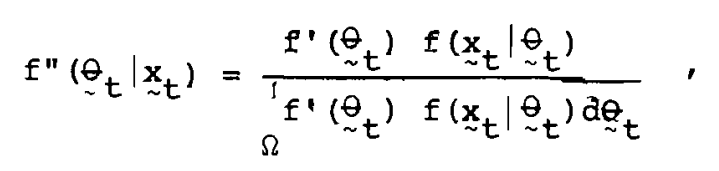


where $\Omega$ is the parameter space. At the start of period $t$, the predictive distribution of $\underset{\sim}{\underset{\mathbf{x}}{f}}$ may be obtained as follows:

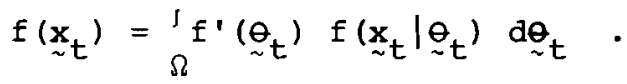

Assume that successive $\underset{\sim t}{\tilde{\theta}}$ are related by

$$
\tilde{\theta}_{t+1}={\underset{\sim}{\partial} t}_{t}+{\underset{\sim}{\tilde{\varepsilon}} t}_{t}
$$

where $\underset{\sim}{\tilde{\varepsilon}}$ is an independent stochastic process with density function $g\left(\varepsilon_{t}\right)$ common to all $t$. (Note that $\underset{\sim}{\tilde{\varepsilon}}$ is not necessarily normally distributed.) In view of (13), the posterior distribution of $\tilde{\theta}_{t}$ may be transformed into a prior distribution of $\tilde{\theta}_{\mathrm{t}+1}$,

$$
f^{\prime}({\underset{\sim t}{\theta}+1})=\int_{\Omega}^{1} f^{\prime \prime}\left(\underset{\sim t}{\theta_{t}} \mid \underset{\sim t}{x_{t}}\right) g\left(\underset{\sim t+1}{\theta_{\tau}} \underset{\sim t}{\theta_{t}}\right) d \underset{\sim t}{\theta_{t}},
$$

and successive $\underset{\sim}{\stackrel{t}{t}}$ can be dealt with in this fashion. Of course, the tractability of the model depends on the specific distributional assumptions. For example, the normal distributions considered earlier in this section yield an especially tractable model.

In this section, it has been demonstrated that the presence of nonstationary means can have an impact upon the uncertainty associated with a given security or set of securities. Moreover, the nonstationary model considered in this section seems to have more realistic properties in this context (forecasting security prices) than the corresponding 
stationary model. For example, in the nonstationary model the recent returns are given more weight than the not-so-recent returns in determining the mean of the distribution at any given time, and the uncertainty about the parameters of the process is never completely removed (since the covariance matrix of $\underset{\sim}{\tilde{\mu}}$ does not approach $\underset{\sim}{\sim}$ as $t$ increases). Of course, the degree of these effects depends upon the degree of the nonstationarity, as measured by the scalar factor $\omega^{-1}$ in the covariance matrix of the shock terms that cause shifts in the mean vector. The model considered here is quite simple, but the properties of the model might be expected to hold under more general conditions, and the same framework can be used to construct forecasting models under much more general conditions of nonstationarity.

\section{A Bayesian Model for Portfolio Selection and Revision}

The model discussed in this section is developed and described in greater detail in Winkler and Barry [18]. In this model portfolio management is viewed as an adaptive process, for sample information is used to update probabilities as more is learned about the properties of the stochastic process generating future security prices. A stationary Bayesian forecasting model is used in [18] to accomplish the probability revision. For simplicity, $m_{t}$ will be used in place of $m_{\sim}^{\prime}$ in this section and the next section to denote the prior mean vector at time $t-1(t=1,2, \ldots)$ of the distribution of $\tilde{\sim}_{t} \cdot$ 
The decision facing the decision maker in the portfolio problem is to determine an optimal portfolio to hold during the first time period. Suppose J securities are under consideration for inclusion in the portfolio, and denote the amount invested in security $j(j=1, \ldots, J)$ at time $t(t=0,1,2, \ldots)$ before portfolio revision by $a_{t}^{j}$. Let $p_{t}^{j}$ and $q_{t}^{j}$ represent the amount of security $j$ that is purchased and sold, respectively, at time $t$. Thus, the amount invested in security $j$ at time $t$ after revision is $a_{t}^{j}+p_{t}^{j}-q_{t}^{j}$. If the rate of return on security $j$ during period $t+1$ is $\check{r}_{t+1}^{j}$, the amount invested in security $j$ at time $t+1$ before revision is

$$
\tilde{a}_{t+1}^{j}=\left(1+\tilde{r}_{t+1}^{j}\right)\left(a_{t}^{j}+p_{t}^{j}-q_{t}^{j}\right)
$$

The problem faced by the decision maker at time $t$ (the end of period $t$ ) is to choose the vectors ${\underset{\sim}{t} t}_{t}=\left\{p_{t}^{j}\right\}$ and $q_{t}=\left\{q_{t}^{j}\right\}$ to maximize $E_{t} U\left(\tilde{W}_{t+1}\right)$, the expected utility of $\tilde{W}_{t+1}=\sum_{j=1}^{j} \tilde{a}_{t+1}^{j}$, the decision maker's wealth at time $t+1$. The subscript on the expectation operator indicates that expectations are taken with respect to the decision maker's probability distribution at time $t$. It is assumed that transactions costs restrict the decision maker's choice of ${\underset{\sim}{t}}_{t}$ and $q_{t}$. In particular, if the amount $z$ of a security is bought or sold, a charge of $\mathrm{cz}$ is levied. Also, short sales are not allowed, and the amount of a security bought or sold is restricted to be nonnegative. 
These considerations lead to the following single-period p.stfolio revision problem:

$\operatorname{maximize} \quad E_{t} U\left[\sum_{j=1}^{J}\left(1+\tilde{r}_{t+1}^{j}\right)\left(a_{t}^{j}+p_{t}^{j}-q_{t}^{j}\right)\right]$

subject to

$$
\begin{aligned}
& \beta \sum_{j=1}^{J} p_{t}^{j} \leq \sum_{j=I}^{J} q_{t}^{j}, \\
& 0 \leq q_{t}^{j} \leq a_{t}^{j}, \quad j=1,2, \ldots, J,
\end{aligned}
$$

and

$$
p_{t}^{j} \geq 0, \quad j=1,2, \ldots, J,
$$

where $\beta=(1+c) /(1-c)$.

Assume that ${\underset{\sim}{\tilde{r}}}_{t+1}$ is normally distributed with unknown mean vector $\tilde{\mu}$ and known covariance matrix $\underset{\sim}{\sum}$ and that the prior distribution of $\underset{\sim}{\tilde{\mu}}$ at $t$ ime $t$ is normal with mean vector $\underset{\sim}{\stackrel{m}{t}+l}$ and covariance matrix $\left(n_{t+1}^{\prime}\right)^{-1} \sum_{\sim}$. This is the model of section 2 under stationarity, and the predictive distribution of ${\underset{\sim}{\tilde{r}}}_{t+1}$ is normal with mean vector ${\underset{\sim}{t+1}}_{t+1}$ and covariance matrix $k_{t+1}{\underset{\sim}{\sim}}_{\text {. }}$.

If the decision maker's utility function for wealth is linear in wealth, the security $i$ with the highest expected return should be purchased and all those securities $j$ such that $\beta\left(1+m_{t+1}^{j}\right) \leqq\left(1+m_{t+1}^{i}\right)$ should be sold. That is, the decision maker should sell those securities for which the transactions costs of selling them and purchasing security $i$ 
will be offset by the greater expected return of $i$. Under a utility function reflecting risk aversion (i.e. $\frac{\mathrm{d}^{2} \mathrm{u}}{\mathrm{dW}^{2}}<0$ ), it is well known that if the distribution of returns is normal an individual will select a portfolio that is efficient in the sense of Markowitz [2]. This implies that the individual will trade off the expected return and variance of various securities in making portfolio selection decisions. In the portfolio revision model presented in this section, the relevant predictive distribution, $\mathrm{f}_{t}\left(\underline{r}_{t+1}\right)$, is normal, so the usual mean-variance tradeoffs are involved. ${ }^{7}$ Because of the presence of transactions costs, however, the gains of a particular revision policy must be traded off against the cost of making the shift. In general, the optimal portfolio revision decision under risk aversion will be a function of each security's expected return, the terms of the covariance matrix $\underset{\sim}{\Sigma}$, the factor $n_{t+1}^{\prime}$ (which reflects the uncertainty about $\underset{\sim}{\tilde{\mu}} t+1$ and affects the scale of the predictive covariance matrix), transactions costs, and the amount of each security held in the portfolio prior to revision.

Two utility functions that reflect risk aversion are the quadratic and exponential functions,

$$
u_{q}(w)=w-b w^{2}, b>0, w<(2 b)^{-1}
$$

and

$$
U_{e}(W)=-\exp (-d w), \quad d>0 .
$$


Under these utility functions, the single-period problem reduces to very simple forms. In particular, consider the problem with only two securities $(J=2)$. If any transaction occurs, one security will be bought and one will be sold. Thus, in view of (17), the amount purchased of one security will be exactly $(1-c) /(1+c)=1 / B$ times the amount sold of the other security:

$$
q_{t}^{i}=B p_{t}^{j}, \quad i=1,2 ; j=1,2 ; i \neq j \text {. }
$$

Under the quadratic and exponential utility functions, the single-period portfolio revision problem reduces to the following simple form:

maximize $k_{1}\left(p_{t}^{1}\right)^{2}+k_{2}\left(p_{t}^{2}\right)^{2}+k_{3}\left(p_{t}^{1}\right)+k_{4}\left(p_{t}^{2}\right)$

subject to

$$
0 \leq p_{t}^{j} \leq \beta^{-1} a_{t}^{j}, \quad j=1,2 \text {. }
$$

The coefficients $K_{1}, K_{2}, K_{3}$, and $K_{4}$ depend upon the particular utility function that is used, and the exact forms of these coefficients under exponential utility are given in section 4 . The solution to the quadratic programming problem in (23) is

$$
\left(p_{t}^{1}, p_{t}^{2}\right)= \begin{cases}\left(\beta^{-1} a_{t}^{2}, 0\right) & \text { if }-K_{3} / 2 K_{1} \geq \beta^{-1} a_{t^{\prime}}^{2} \\ \left(-K_{3} / 2 K_{1}, 0\right) & \text { if } 0 \leq-K_{3} / 2 K_{1}<\beta^{-1} a_{t^{\prime}}^{2} \\ (0,0) & \text { if }-K_{3} / 2 K_{1}<0 \text { and }-K_{4} / 2 K_{2}<0 \\ \left(0,-K_{4} / 2 K_{2}\right) & \text { if } 0 \leq-K_{4} / 2 K_{2}<\beta^{-1} a_{t^{\prime}}^{1} \\ \left(0, \beta^{-1} a_{t}^{1}\right) & \text { if }-K_{4} / 2 K_{2} \geq \beta^{-1} a_{t}^{1}\end{cases}
$$


and this provides the optimal portfolio revision policy for this situation.

In this section a very brief sketch of a Bayesian model for portfolio choice has been presented. Of course, the model could easily be generalized, and more details are given in [18]. For example, although only a single-period model has been considered here, a multiperiod adaptive portfolio model is considered in some detail in [18].

\section{Nonstationarity and Portfolio Choice}

In Section 2, a Bayesian model for forecasting future security prices under nonstationary means was presented, and it was compared with a related stationary model. The results of applying the stationary model in a portfolio revision context were described in Section 3. In this section, the implications of nonstationarity for portfolio choice will be considered through the application of the nonstationary forecasting model to the portfolio revision problem.

In the portfolio model of Section 3 , the probability distribution of interest at time $t$ is the predictive distribution of $\underset{\sim t+1}{\tilde{r}_{t+1}}$. In the forecasting model of section 2, this predictive distribution is normal with mean vector $\operatorname{m}_{\sim t+1}^{\prime}$ and covariance matrix $k_{t+1} \sum_{\sim}$, where $k_{t+1}=\left(n_{t+1}^{\prime}+1\right) / n_{t+1}^{\prime}$. The predictive distribution under nonstationarity is identical to the distribution that would be obtained under stationarity except that $k_{t+1}$ is larger in the case of nonstationary means than in the case of stationary means. Hence, something can be learned about the effects of nonstationarity upon portfolio 
choice through an analysis of the effects of a change in $k_{t+1}$ upon portfolio choice.

\section{Linear Utility}

In the case of linear utility, the optimal portfolio revision policy at time $t$ depends only upon $\underset{\sim}{\mathrm{m}} \mathrm{t+1}$ and $\beta$. Hence, since the only short-run effect of nonstationarity of the form considered in this section is to change $k_{t+1}$, decisions made on the basis of linear utility are unaffected by nonstationarity in the short run.

The limiting case is quite different, however. As indicated in Section 2, under stationarity all returns are weighted equally and $\mathrm{m}_{\sim t}$ converges to $\underset{\mu}{\mu}$ as $t$ tends to infinity. Thus, under the decision rule for linear utility, there is only an infinitesimal probability that any security purchases and sales will be made after sufficiently long $t$ because $\underset{\sim t}{m}$ will remain virtually unchanged from period to period. Under nonstationarity, however, regardless of how large $t$ is, recent returns are given more weight than not-so-recent returns, and $m_{t}$ may change substantially from one period to the next as $\tilde{\sim}_{t}$ changes. Thus, some purchases and sales may be optimal at any point in time. In effect, the decision maker's evaluation of any security may continue to change regardless of how long the decision maker has been following that security.

\section{Risk Aversion and A Single Risky Security}

Nonstationarity has no short-run effect on decisions made via a linear decision rule in a one-period framework, but such is 
not the case when the decision maker is risk-averse. For the sake of illustration, consider problem (23) under exponential utility. The optimal policy is given by (25) with

$$
\begin{aligned}
& \mathrm{k}_{1}=-d \mathrm{k}_{t+1}\left(\sigma_{1}{ }^{2}-2 \beta \sigma_{12}+\beta^{2} \sigma_{2}{ }^{2}\right) / 2, \\
& \mathrm{k}_{2}=-d \mathrm{k}_{t+1}\left(\beta^{2} \sigma_{1}{ }^{2}-2 \beta \sigma_{12}+\sigma_{2}{ }^{2}\right) / 2, \\
& \mathrm{k}_{3}=\left(1+\mathrm{m}_{t+1}^{1}\right)-\beta\left(1+\mathrm{m}_{t+1}^{2}\right)-d k_{t+1}\left[\mathrm{a}_{t}^{1} \sigma_{1}{ }^{2}\right. \\
&\left.+\left(a_{t}^{2}-\beta a_{t}^{1}\right) \sigma_{12}-\beta a_{t}^{1} \sigma_{2}{ }^{2}\right],
\end{aligned}
$$

and

$$
\begin{aligned}
\mathrm{k}_{4}=\left(1+\mathrm{m}_{t+1}^{2}\right) & -\beta\left(1+\mathrm{m}_{t+1}^{1}\right)-d \mathrm{k}_{t+1}\left[-\beta \mathrm{a}_{t}^{1} \sigma_{1}^{2}\right. \\
& \left.+\left(\mathrm{a}_{t}^{1}-\beta \mathrm{a}_{t}^{2}\right) \sigma_{12}+\mathrm{a}_{t}^{1} \sigma_{2}^{2}\right],
\end{aligned}
$$

where

$$
\Sigma=\left[\begin{array}{ll}
\sigma_{1}^{2} & \sigma_{12} \\
\sigma_{12} & \sigma_{2}^{2}
\end{array}\right] .
$$

Furthermore, let security one be risk-free (i.e. $m_{t+1}^{1}=r_{t+1}^{1}$ and $\sigma_{1}^{2}=\sigma_{12}=0$ ).

From (25) it is apparent that the effects of a change in one of the parameters of the problem can be analyzed by considering the effects of the change upon $-\mathrm{K}_{3} / 2 \mathrm{~K}_{1}$ and $-\mathrm{K}_{4} / 2 \mathrm{~K}_{2}$. Since the effect of nonstationarity is to increase $k_{t+1}$, thereby increasing the predictive variance of the return of the risky security, the partial derivatives 


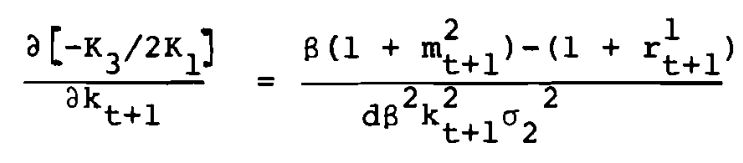

and

$$
\frac{\partial\left[-k_{4} / 2 k_{2}\right]}{\partial k_{t+1}}=\frac{B\left(1+r_{t+1}^{1}\right)-\left(1+m_{t+1}^{2}\right)}{d k_{t+1}^{2} \sigma_{2}^{2}}
$$

are of interest. The derivative in (31) is positive under the sufficient (but not necessary) condition that the expected return on the risky security exceeds that of the risk-free security. The derivative in (32) is negative if $\left(1+\mathrm{m}_{t+1}^{2}\right)$ $>B\left(1+r_{t+1}^{1}\right)$, which is a necessary condition for the risky security to be purchased. Hence, the amount of the risky security held tends to decrease as nonstationarity is introduced.

Thus, the short-run effect of nonstationarity in the case of a single risky security competing with a riskless asset (which is equivalent to the case of a given portfolio competing with a riskless asset) under exponential utility is to make the risky security less attractive. This is because the central effect of the nonstationarity is to increase the variance of the return from the risky security while leaving its expected return unchanged. This result also holds in the case of quadratic utility, and it should be expected to hold for any utility function implying aversion to risk. The long-run effect of nonstationarity in the case of a single risky security and a risk-averse decision maker is identical to the 
long-run effect in the linear utility case; portfolio revision may be optimal even for large $t$, since the decision maker's evaluation of the risky security may continue to change regardless of how long the decision maker has been following that security.

\section{Risk Aversion and Two Risky Securities}

When the two securities competing for investment dollars are both risky, the effect of nonstationarity upon an optimal allocation should be dependent upon the characteristics of both of the securities. Once again, the effects of the increase in $k_{t+1}$ caused by nonstationarity can be investigated by finding the partial derivatives of $-\mathrm{K}_{3} / 2 \mathrm{~K}_{1}$ and $-\mathrm{K}_{4} / 2 \mathrm{~K}_{2}$ with respect to $\mathrm{k}_{t+1}$. Moreover, since both securities are risky, the problem is symmetric, and it is on $l_{y}$ necessary to consider the derivative of $-\mathrm{K}_{3} / 2 \mathrm{~K}_{1}$. The results for the case of exponential utility are presented here, based on the coefficients in (26)-(29). (Results under quadratic utility, which are not presented here, are similar to the findings under exponential utility.)

In the case of exponential utility,

$$
\begin{aligned}
\frac{\partial\left[-\mathrm{k}_{3} / 2 \mathrm{~K}_{1}\right]}{\partial \mathrm{k}_{\mathrm{t}+1}}= & \mathrm{d}\left[\beta\left(1+\mathrm{m}_{\mathrm{t}+1}^{2}\right)-\left(1+\mathrm{m}_{\mathrm{t}+1}^{1}\right)\right] \\
& \cdot\left(\sigma_{1}{ }^{2}-2 \sigma_{12}+\beta^{2} \sigma_{2}{ }^{2}\right) / 4 \mathrm{~K}_{1}{ }^{2} .
\end{aligned}
$$

Since $\beta \geq 1$ and $\left|\rho_{12}\right| \leq 1$, where $\rho_{12}=\sigma_{12} / \sigma_{1} \sigma_{2}$ is the correlation coefficient, $\sigma_{1}^{2}-2 \sigma_{12}+B^{2} \sigma_{2}{ }^{2}>0$. Thus, the quali- 
tative effects of nonstationarity in this case, as measured by the sign of the derivative in (33), depend only upon the expected returns of the two securities and upon $\beta$ (an index reflecting transactions costs), although the actual amounts purchased and sold also depend upon $k_{t+1}$ and $\underset{\sim}{\Sigma}$.

From (33), it follows that if some amount (but not the largest possible amount) of security one should be purchased under stationarity (i.e. if $0<-\mathrm{K}_{3} / 2 \mathrm{~K}_{1}<\beta^{-1} \mathrm{a}_{t}^{2}$ ), then less of that security should be purchased under nonstationarity if

$$
1+m_{t+1}^{1}>\beta\left(1+m_{t+1}^{2}\right),
$$

more of that security should be purchased if the inequality is reversed, and the same amount should be purchased if equality holds. Given that it is optimal to purchase some of security one, then, the amount purchased increases as the expected return from security one decreases relative to the expected return from security two. ${ }^{8}$ An intuitive explanation can be offered for this somewhat surprising result. For example, suppose that under stationarity some amount of security one should be purchased but that (34) does not hold (i.e. security one is unattractive to a risk-neutral investor). Then in order for a risk-averse individual to buy the security it must offer some reduction in variance for the portfolio. Under nonstationarity the variance of each portfolio is greater, so variance-reduction becomes even more important to the riskaverse investor. Hence, more of the security promising lower portfolio variance should be purchased. On the other hand, if 
some amount of security one should be purchased under stationarity and (34) holds, then less of security one should be purchased under nonstationarity. This result is intuitively appealing only if purchasing security one increases the portfolio variance (i.e. if security one is purchased because of a high expected return despite a high variance), which would make security one less attractive in the presence of nonstationarity.

Thus, the short-run effect of nonstationarity in the case of two risky securities under exponential utility depends upon the expected returns from the two securities, and the sort of tradeoffs between expected return and variance of return that have been discussed in the context of two risky securities should also hold in problems involving more than two risky securities. Once again, the long-run effect of nonstationarity is identical to the long-run effect in the linear utility case.

\section{Nonstationarity and Traditional Portfolio Analysis}

The bulk of single-period portfolio analysis in recent years has dealt with the Markowitz portfolio selection model [2] or some variation thereof. The Markowitz model deals with finding an efficient set of portfolios (i.e. the set of all portfolios having minimum variance for given levels of expected return or having maximum expected return for given levels of variance). In this section this model will be considered in view of the results in section 2 concerning forecasting under nonstationarity. 
Since the only short-run difference between the stationary and nonstationary models considered here is that the covariance matrix is increased by a scalar factor in the nonstationary case, the effects of nonstationarity may be examined by merely seeing how a scalar increase in the covariance matrix affects the efficient set and the choice of an optimal portfolio. In studying the somewhat related problem of the effects of unknown (but stationary) parameters upon mean-variance portfolio analysis, Barry [22] found that under assumptions similar to those used here, the covariance matrix of returns was multiplied by a scalar larger than one. Hence, the initial effect was the same as that found here for nonstationarity, and therefore the analysis in that paper is directly relevant to the current problem.

Nonstationarity leaves the efficient set unchanged in the sense that all portfolios that are efficient under stationarity remain efficient under nonstationarity. However, a map of the efficient set in mean-variance space shows that the set is shifted to the right (following the convention of placing variance on the horizontal axis). This implies that the optimal package of risky securities (i.e. the optimal portfolio excluding the risk-free security) changes. The change is to a package with lower expected return, implying a less risky package as well (see [22] for details).

In short, as uncertainty is increased via nonstationarity, the perceived risk of each portfolio increases. This causes the investor to "retreat" to a less risky package. This result is consistent with the earlier findings in this section in 
which the effects of nonstationarity were considered in the context of a single-period version of a Bayesian model for portfolio selection and revision.

\section{Summary and Discussion}

In this paper, a Bayesian model for forecasting future security prices under nonstationarity has been described and compared with a corresponding stationary model. In terms of the short-run behavior of the models, greater uncertainty is retained under nonstationarity than under stationarity. In terms of the limiting behavior of the models, the values of the parameters of interest cannot be ascertained with certainty under nonstationarity, even after the process has been observed for many time periods, and any given observed returns receive less weight as the length of time since the observed returns increases. These properties are not shared by the corresponding stationary model, and in general, the nonstationary model considered in this paper appears to have more realistic properties than the corresponding stationary model.

With respect to portfolio choice under linear utility, nonstationarity has no effect in the short run but may prevent the curtailment of trading in the long run that occurs under the stationary model. For a risk-averse decision maker considering one risky security and one risk-free security, nonstationarity decreases the attractiveness of the risky security. This implies that in general, a risk-averse decision maker will invest less money in a portfolio of risky securi- 
ties in the nonstationary case than in the stationary case. When the two securities under consideration are both risky, the effect of nonstationarity for a risk-averse decision maker can be related to the expected returns for the two securities. With respect to traditional mean-variance analysis, nonstationarity does not affect the membership of the efficient set of portfolios, but the efficient set does shift in mean-variance space due to the additional uncertainty under nonstationarity, and this causes a change in the optimal portfolio.

Various extensions of the forecasting model could be considered, and the portfolio selection and revision model could be reexamined in the light of such extensions. In view of recent empirical support for nonstationary variance terms in stock price distributions, the analysis of the effects of nonstationary variances and covariances on portfolio choice would be a logical extension of the analysis in this paper. Winkler [17] considered the case of an unknown covariance matrix, and that approach could be extended to include a nonstationary covariance matrix. Some additional extensions that might add to the realism of the analysis in this paper include the consideration of the case in which the process generating changes in the mean vector is characterized by unknown parameters, although the model could become quite cumbersome as additional uncertainty is Introduced. (Also, as the number of unknown parameters is increased, the identifiability of the model may become a problem, as the data may not permit inferences about all of the parameters of interest.) Another possible exten- 
sion is to consider the case in which changes in the unknown parameters occur at random intervals of time rather than at fixed intervals of time. Carter [23] considered such an extension for the univariate situation studied by Bather [24], and it appears to add considerable realism to the model. However, analytical results for that case may be difficult to obtain.

Nonstationarity has long been neglected in the study of economic decision models in general and in the study of portfolio analysis in particular. Although the results of this paper are obtained under a relatively simple model, the point is that nonstationarity can have effects on portfolio decisions and hence upon the functioning of capital markets. Further work of both an empirical and analytical nature concerning the existence of and effects of nonstationarity appears warranted. 


\section{Footnotes}

${ }^{1}$ Although nonstationarity has been neglected in the study of economic decision models, some recent work in econometrics has dealt with inferential problems of time-varying parameters in regression analysis, and recently a special issue of the Annals of Economic and Social Measurement was devoted exclusively to that topic (see Rosenberg [1] for a review of literature in this area).

2 A tilde over a variable indicates that it is a random variable, and vectors and matrices are shown in boldface.

${ }^{3} \mathrm{~A}$ normal prior distribution of $\tilde{\mu}$ is conjugate with respect to sampling from a normal data-generating process with unknown mean vector $\tilde{u}$ and known covariance matrix $\sum_{\sim}$, although it is not required that the prior covariance matrix be a scalar multiple of $\sum_{\sim}$. See Raiffa and Schlaifer [19] for a discussion of conjugate families of distributions in Bayesian inference.

${ }^{4}$ In the presence of drift, the limiting expected price differences would be infinite, which seems unreasonable. Thus, assuming $\mathbf{e}=0$ appears warranted.

5 Under these assumptions, variance (or standard deviation) provides a valid measure of risk. For a discussion of this point, see Tobin [21].

${ }^{6}$ Because the prior and posterior distributions of $\tilde{\mu}_{t}$ during time period $t$ have been assumed to be identical for the stationary and nonstationary models, the difference between the values of $k_{t+1}$ in the two models reflects only the impact of a single period of nonstationarity. If several periods are considered, the impact will of course be much greater.

\section{${ }^{7}$ Because of the treatment of $\tilde{\mu}$ as a random variable in} the Bayesian forecasting model and because this treatment may be extended to include $\sum_{\sim}$, the optimal portfolio will not necessarily be the same as that derived under the assumption that $\tilde{\mu}$ and $\tilde{\tilde{\Sigma}}$ are known. The qualitative results are similar, but the spec̃lfics differ, even if transactions costs are ignored (see [22]).

\footnotetext{
${ }^{8}$ of course, this is only a "local" effect; as $m_{t+1}^{1}$ decreases relative to $\mathrm{m}_{\mathrm{t}+1}^{2}$, eventually the sign of $-\mathrm{K}_{3} / 2 \mathrm{~K}_{1}$ will change and security one will no longer be purchased at all.
} 
References

[1] Rosenberg, Barr, "A survey of Stochastic Parameter Regression," Annals of Economic and Social Measurement, 2 (1973), 381-397.

[2] Markowitz, Harry M., Portfolio Selection: Efficient Diversification of Investments. New York: Wiley, 1959.

[3] Francis, Jack Clark, and Archer, Stephen H, , Portfolio Analysis. Englewood Cliffs, New Jersey: PrenticeHa11, 1971 .

[4] Tobin, James, "The Theory of Portfolio Selection," in F. H. Hahn and F.P.R. Brechling (eds.), The Theory of Interest Rates. New York: Macmillan, 1965.

[5] Mossin, Jan, "Optimal Multiperiod Portfolio Policies," Journal of Business, 41 (1968), 215-229.

[6] Chen, Andrew H. Y., Jen, Frank C., and Zionts, Stanley, "The Optimal Portfolio Revision Policy," Journal of Business, 44 (1971), 51-61.

[7] Hakansson, Nils H., "Multiperiod Mean-Variance Analysis: Toward a General Theory of Portfolio Choice," Journal of Finance, 26 (1971), 857-884.

[8] Hakansson, Nils H., "Optimal Consumption Investment Strategies Under Risk for a Class of Utility Functions," Econometrica, 38 (1970), 587-607.

[9] Samuelson, Paul A., "Lifetime Portfolio Selection By Dynamic Stochastic Programming," Review of Economics and Statistics, 51 (1969), 239-2 $\overline{46 .}$

[10] Fama, Eugene F., "Multiperiod Consumption-Investment Decisions," American Economic Review, 60 (1970), 163-174.

[11] Meyer, Richard F., "On the Relationship Among the Utility Assets, the Utility of Consumption, and Investment Strategy in an Uncertain but Time-Invariant World," in Proceedings of the Fifth International Conference on Operational Research, London: Tavistock, (1970), 627-648. 
[12] Barnea, Amir, and Downes, David H., "A Reexamination of the Empirical Distribution of Stock Price Changes," Journal of the American Statistical Association, $68(1973), 348-350$.

[13] Praetz, Peter D., "The Distribution of Share Price Changes," Journal of Business, 45 (1972), 49-55.

[14] Blattberg, R. C., and Gonedes, N. J., "A Comparison of the Stable and student Distributions as Statistical Models for Stock Prices," Journal of Business, 47 $(1974), 244-280$.

[15] Boness, A. James, Chen, Andrew H., and Jatusipitak, Som, "A Hypothesis of Nonstationary Common Share Price Changes," unpublished manuscript, State University of New York at Buffalo, 1972.

[16] Hsu, Der-Ann, Miller, Robert B., and Wichern, Dean W., "On the Stable Paretian Behavior of Stock Market Prices," Journal of the American Statistical Association, $69(1974), 108-113$.

[17] Winkler, Robert L,, "Bayesian Models for Forecasting Future Security Prices," Journal of Financial and Quantitative Analysis, 8 (1973), 387-405.

[18] Winkler, Robert L., and Barry, Christopher B., "A Bayesian Model for Portfolio Selection and Revision," Journal of Finance, in press, 1973.

[19] Raiffa, Howard, and Schlaifer, Robert, Applied Statistical Decision Theory. Boston: Graduate School of Business Administration, Harvard University, 1961.

[20] Winkler, Robert L., and Barry, Christopher B., "Nonstationary Means in a Multinormal Process," Research Report RR-73-9, International Institute for Applied Systems Analysis, Laxenburg, Austria. 1973.

[21] Tobin, James A., "Liquidity Preference as Behavior Towards Risk," Review of Economic Studies, 26 (1958), 65-86.

[22] Barry, Christopher B., "Portfolio Analysis Under Uncertain Means, Variances, and Covariances," Journal of Finance, 29 (1974), 515-522.

[23] Carter, Phillip L., "A Bayesian Approach to Quality Control," Management Science, 18 (1972), 647-655.

[24] Bather, J. A., "Control Charts and the Minimization of Costs," Journal of the Royal Statistical Society Series B, 25 (1963), 49-80. 